\title{
Association of aortic valve calcification with carotid artery lesions and peripheral artery disease in patients with chronic kidney disease: a cross- sectional study
}

\section{Yui Arita}

Division of Nephrology, Department of Internal Medicine, Yamaguchi Red Cross Hospital and Division of Nephrology and Clinical Research Institute, Department of Internal Medicine, National Hospital Organization Kyushu Medical Center

\section{Masaru Nakayama ( $\sim$ nakayama51224@gmail.com)}

Division of Nephrology and Clinical Research Institute

\section{Yuta Matsukuma}

Division of Nephrology and CInical Research Institute, Department of Internal Medicne, National Hospital Organization Kyushu Medical Center

\section{Ryota Yoshitomi}

Division of Nephrology and Clinical Research Institute, Department of Internal Medicine, National Hospital Organization Kyushu Medical Center

\section{Makiko Seki}

Division of Nephrology and Clinical Research Institute, Department of Internal Medicine, National Hospital Organization Kyushu Medical Center Akiko Fukui

Divsion of Nephrology and Clinical Research Institute, Department of Internal Medicine, National Hospital Organization Kyushu Medical Center Susumu Tsuda

Division of Nephrology and Clinical Research Institute, Deparment of Internal Medicine, National Hospital Organization Kyushu Medical Center Yuri Sonoda

Division of Nephrology and Clinical Research Institute, Deparment of Internal Medicine, National Hospital Organization Kyushu Medical Center

Rina Imazu

Division of Hypertension and Clinical Research Institute, Department of Internal Medicine, National Hospital Organization Kyushu Medical Center

Kimika Arakawa

Division of Hypertension and Clinical Research Institute, Department of Internal Medicine, National Hospital Organization Kyushu Medical Center Mitsuhiro Tominaga

Division of Hypertension and Clinical Research Institute, Depaerment of Internal Medicine, National Hospital Organization Kyushu Medical Center

Toshiaki Nakano

Department of Medicine and Clinical Science, Graduate School of Medical Sciences, Kyushu University

Kazuhiko Tsuruya

Department of Nephrology, Nara Medical University

Takanari Kitazono

Department of Medicine and Clinical Science, Graduate School of Medical Sciences, Kyushu University

\section{Research article}

Keywords: Aortic valve calcification, Atherosclerosis, Carotid artery plaque, Chronic kidney disease, Peripheral artery disease.

Posted Date: April 23rd, 2020

DOI: https://doi.org/10.21203/rs.3.rs-24088/v1

License: (c) (i) This work is licensed under a Creative Commons Attribution 4.0 International License. Read Full License

Version of Record: A version of this preprint was published at BMC Nephrology on May 29th, 2020. See the published version at https://doi.org/10.1186/s12882-020-01864-z. 


\section{Abstract}

Background: Patients with chronic kidney disease (CKD) reportedly have a high prevalence of aortic valve calcification (AVC). In population-based studies, AVC is considered a manifestation of systemic atherosclerosis. The association of AVC with atherosclerotic lesions has not been fully investigated in predialysis patients. The present study was performed to determine whether carotid artery lesions and peripheral artery disease (PAD) are associated with AVC in patients with CKD not on dialysis.

Methods: In total, 749 patients were included in this cross-sectional study. AVC was evaluated using echocardiography. Carotid artery lesions including carotid artery plaque (CAP) and PAD were simultaneously examined in each patient. A logistic regression analysis was applied to determine the factors associated with AVC.

Results: AVC, CAP, and PAD were found in 201, 583, and 123 patients, respectively. In the multivariable analyses adjusted for covariates including the estimated glomerular filtration rate and makers of mineral metabolism (serum calcium, serum phosphorus, parathyroid hormone, 1,25-dihydroxyvitamin D, and fibroblast growth factor 23), AVC was significantly associated with the presence of CAP [odds ratio (OR), 3.37; 95\% confidence interval (CI), 1.43-7.95], the presence of PAD (OR, 1.76; $95 \% \mathrm{Cl}, 1.10-2.81)$, the CAP score (per 1.0-point increase) $(\mathrm{OR}, 1.06 ; 95 \% \mathrm{Cl}, 1.02-1.11)$, and the ankle-brachial blood pressure index (per 0.1-point increase) (OR, 0.83; 95\% Cl, 0.72-0.95).

Conclusions: AVC was associated with atherosclerotic lesions independent of kidney function and mineral metabolism. We consider that this association between AVC and atherosclerosis might reflect the burden of shared atherosclerotic risk factors.

\section{Background}

Patients with chronic kidney disease (CKD) are considered to have a high risk of cardiovascular (CV) morbidity and mortality [1]. A previous study showed that left-sided valve disease such as aortic stenosis and mitral regurgitation was highly prevalent and related to higher mortality in patients with CKD [2]. Mortality due to $\mathrm{CV}$ disease is 10-30-fold higher in dialysis patients than in the general population [3]. In addition, in predialysis patients, the risk of all-cause mortality increased as estimated glomerular filtration rate (eGFR) decreased, in one study, especially in patients with eGFR $<15 \mathrm{~mL} / \mathrm{min} / 1.73 \mathrm{~m}{ }^{2}$, who had a 5.9 -fold increase in the risk of all-cause mortality compared with that in patients with eGFR $\geq 60 \mathrm{~mL} / \mathrm{min} / 1.73 \mathrm{~m}^{2}$ [4]. Research has demonstrated that moderate/severe aortic valve calcification (AVC) is associated with CV events and mortality in patients with aortic stenosis $[5,6]$ and in the general population $[7,8]$. Furthermore, in dialysis [9] or predialysis [10] patients, univariable analysis showed a significant association of AVC with all-cause mortality; however, multivariable analysis did not.

The reported prevalence of AVC ranges from $6.6-18.4 \%$ in population-based studies $[8,11-16]$. In contrast, patients with CKD have been shown to have a high prevalence of AVC. In dialysis patients, the prevalence of AVC ranges from 14.1-75.0\% [9, 17-20], while predialysis patients with CKD show a prevalence of $18.6-36.5 \%$ [10, 21-23]. Many studies to date have explored the factors associated with AVC. Traditional CV risk factors such as hypertension, diabetes mellitus, smoking, dyslipidemia, male sex, and older age, similar to the risk factors for atherosclerosis, have been associated with AVC in population-based studies [15, 24]. In patients with CKD, Leskinen et al. [25] and Wang et al. [18] demonstrated that a combination of aortic and mitral valve calcification was associated with carotid artery lesions including carotid artery plaque (CAP) and peripheral artery disease (PAD), which are recognized as surrogate markers of subclinical atherosclerosis [26, 27]. The study participants were predialysis, dialysis, and renal transplantation patients in the former report and dialysis patients in the latter report. In addition, among dialysis patients, one study showed that the carotid intima-media thickness (IMT) was significantly higher in patients with than without AVC [20]. Nevertheless, whether an association of AVC with carotid artery lesions and PAD exists in predialysis patients remains unknown. Furthermore, especially in patients with CKD, in addition to traditional CV risk factors, nontraditional CV risk factors such as renal dysfunction itself and deranged mineral metabolism [e.g., hyperphosphatemia, elevated parathyroid hormone (PTH) or fibroblast growth factor 23 (FGF23)] associated with CKD are also associated with AVC [28-32]. However, in predialysis patients, the association of AVC with carotid artery lesions and PAD beyond both traditional and nontraditional CV risk factors remains uncertain. Thus, we performed the present study to elucidate whether both carotid artery lesions and PAD are associated with AVC independent of traditional and nontraditional CV risk factors associated with CKD in patients with stage 1-5 CKD not receiving dialysis.

\section{Methods}

\section{Patients and study design}

In total, 821 patients were admitted to our hospital for evaluation and education of CKD from May 2010 to July 2019. We excluded 72 patients who underwent aortic valve replacement $(n=7)$ or had no available data regarding echocardiography $(n=7)$, blood samples $(n=33)$, ankle-brachial blood pressure index (ABPI) $(n=5)$, or carotid artery lesions $(n=20)$. The remaining 749 patients were enrolled in this cross-sectional study, and no patients had bicuspid aortic valves or rheumatic heart disease.

Blood samples were obtained from each patient in the early morning after an overnight fast. Daily proteinuria was also measured. The intact FGF23 level was measured using an FGF23 enzyme-linked immunosorbent assay kit (Kainos Laboratories Inc., Tokyo, Japan). The intact PTH level was measured by electrochemiluminescence immunoassay (Elecsys PTH; Roche Diagnostics GmbH, Mannheim, Germany). A radioimmunoassay was used to measure the 1,25-dihydroxyvitamin D [1,25(OH) 2 D] level (Immunodiagnostic Systems Limited, Boldon, UK). The eGFR ( $\left.\mathrm{mL} / \mathrm{min} / 1.73 \mathrm{~m}^{2}\right)$ was calculated using the new Japanese equation: eGFR $=194 \times \mathrm{SCr}^{-1.094} \times$ age $^{-0.287} \times 0.739$ (if female) [33], where SCr is the serum creatinine level. CKD was defined as abnormal kidney structure or function that persisted for $>3$ months, as indicated by eGFR $<60 \mathrm{~mL} / \mathrm{min} / 1.73 \mathrm{~m}^{2}$ or by kidney damage regardless of a decrease in eGFR, in accordance with the Kidney Disease: Improving Global Outcomes clinical practice guidelines [34]. 
All enrolled patients were interviewed and clinically examined at presentation. Their medical histories and outpatient records were evaluated in detail. Demographic information (age and sex), medication history, and atherosclerotic risk factors (hypertension, history of smoking, dyslipidemia and diabetes mellitus) at presentation were recorded for each patient. Hypertension was defined as systolic blood pressure of $\geq 140 \mathrm{mmHg}$, diastolic blood pressure of $\geq$ $90 \mathrm{mmHg}$, or the current use of antihypertensive drugs. Diabetes mellitus was defined as a previous or current plasma fasting glucose level of $\geq 6.99 \mathrm{mmol} / \mathrm{L}$ or the use of hypoglycemic agents. Dyslipidemia was defined as plasma triglycerides $\geq 1.69 \mathrm{mmol} / \mathrm{L}$, plasma low-density lipoprotein cholesterol $\geq$ $3.62 \mathrm{mmol} / \mathrm{L}$, plasma high-density lipoprotein cholesterol < $1.03 \mathrm{mmol} / \mathrm{L}$, or the use of lipid-lowering drugs based on a history of dyslipidemia. Ischemic heart disease was defined as a history of angina, myocardial infarction, coronary angioplasty, or coronary artery bypass surgery. Cigarette smoking was evaluated as current or past. The body mass index was calculated as weight in $\mathrm{kg}$ divided by height in $\mathrm{m}^{2}$. Blood pressure was measured on the second day of hospitalization at three separate times, with the patient in a sitting position; the average of the three readings was recorded.

The method of ABPI measurement is described in our previous report [35]. PAD was defined as having a low ABPI $(<0.9)$ [36] or undergoing interventions for lower limb ischemia. In addition, we examined the carotid IMT using a 5- to 12-MHz linear array transducer with a high-resolution B-mode scanning protocol, as previously described [37]. The CAP score was calculated by summing the thickness of all plaques in the bilateral carotid bifurcation and the bilateral common and internal carotid arteries. Echocardiography was performed simultaneously in each patient. The definition of left ventricular hypertrophy is described in our previous report [38]. The presence or absence of AVC was determined visually and only qualitatively using echocardiography, and the number of calcified aortic cusps was examined.

\section{Statistical analysis}

Continuous data are expressed as either mean \pm SD or median (interquartile range), depending on their distribution. Categorical data are expressed as $\mathrm{n}$ (\%). The non-normally distributed C-reactive protein, FGF23, PTH, and 1,25(OH) ${ }_{2} \mathrm{D}$ levels were log-transformed to achieve an approximately normal distribution prior to statistical analysis. A logistic regression model was also applied to elucidate the factors associated with AVC. The odds ratio (OR) and $95 \%$ confidence interval $(\mathrm{Cl})$ were calculated for each variable. In addition, we examined linear regression analyses to evaluate the association between AVC score and CAP score, ABPI, and eGFR. All statistical analyses were performed using STATA ver. 14 (StataCorp. 2015. Stata Statistical Software. Release 14. College Station, TX: StataCorp LP.). A $P$-value of $<0.05$ was considered to indicate statistical significance.

\section{Results}

The median age of the 749 patients (499 men and 250 women) in this study was 70 years (range, 20-95 years). The primary causes of renal disease were chronic glomerulonephritis (29.1\%, 218 patients), hypertensive nephrosclerosis ( $28.7 \%, 215$ patients), diabetic nephropathy (21.9\%, 164 patients), other defined causes (17.5\%, 131 patients), and unknown causes (2.8\%, 21 patients). Of the total 749 patients, 127 (17.0\%), 221 (29.5\%), 237 (31.6\%), and 164 (21.9\%) patients were categorized as having stage $1-2,3,4$, and 5 CKD, respectively. The prevalence of AVC was $26.8 \%$ (201 patients). CAP and PAD were found in 583 and 123 patients, respectively. The prevalence of AVC in patients with stage $1-2,3,4$, and 5 CKD was $11.0 \%, 25.3 \%, 30.8 \%$, and $35.4 \%$, respectively $(P$ for trend $<0.01$ ). Table 1 shows the clinical characteristics of patients with and without AVC. Patients with AVC were divided into three groups according to the number of calcified aortic cusps. Patients of older ages experienced a higher prevalence of AVC. In patients with AVC, the prevalence of hypertension, diabetes mellitus, ischemic heart disease, and left ventricular hypertrophy was significantly higher while the eGFR and serum albumin level were significantly lower than those in patients without AVC. Pack-years of smoking was higher in patients with AVC. With regard to the association of AVC with mineral metabolism, a lower $1,25(\mathrm{OH})_{2} \mathrm{D}$ level and a higher FGF23 level were related to AVC. AVC was significantly associated with the presence of CAP and PAD, a higher CAP score, and a lower ABPI. In addition, linear regression analyses showed that AVC score was related to CAP score (supplementary material 1 ), ABPI (supplementary material 2 ), and eGFR (supplementary material 3). 
Table 1

Clinical characteristics of patients with and without AVC

\begin{tabular}{|c|c|c|c|c|c|c|}
\hline \multirow[b]{2}{*}{ Variables } & \multirow[t]{2}{*}{ All patients } & \multirow[t]{2}{*}{$\operatorname{AVC}(-)$} & \multicolumn{3}{|l|}{$\operatorname{AVC}(+)(n=201)$} & \multirow[t]{3}{*}{${ }^{*} P$ for trenc } \\
\hline & & & 1 aortic cusp & 2 aortic cusps & 3 aortic cusps & \\
\hline & $(n=749)$ & $(n=548)$ & $(n=116)$ & $(n=47)$ & $(n=38)$ & \\
\hline Age (years) & $70(59-78)$ & $66(53-75)$ & $76(69-80)$ & $80(74-84)$ & $80(74-83)$ & $<0.01$ \\
\hline Male, n (\%) & 499 (67) & $357(65)$ & $80(69)$ & $35(74)$ & $27(71)$ & 0.15 \\
\hline Hypertension, n (\%) & $617(82)$ & $433(79)$ & $102(88)$ & $44(94)$ & $38(100)$ & $<0.01$ \\
\hline Diabetes mellitus, n (\%) & $283(38)$ & $191(35)$ & $51(44)$ & $24(51)$ & $17(45)$ & 0.01 \\
\hline Smoking, n (\%) & $406(54)$ & $287(52)$ & $68(59)$ & $29(62)$ & $22(58)$ & 0.14 \\
\hline Pack-years of smoking ${ }^{\star \star}$ & $6(0-40)$ & $2(0-37)$ & $20(0-48)$ & $30(0-50)$ & $9(0-56)$ & $<0.01$ \\
\hline IHD, n (\%) & $109(15)$ & $63(12)$ & $21(18)$ & $8(17)$ & $17(45)$ & $<0.01$ \\
\hline Dyslipidemia, n (\%) & $552(74)$ & $404(74)$ & $88(76)$ & $33(70)$ & $27(71)$ & 0.70 \\
\hline SBP (mmHg) & $133 \pm 18$ & $132 \pm 18$ & $136 \pm 17$ & $136 \pm 19$ & $135 \pm 17$ & $<0.01$ \\
\hline $\mathrm{DBP}(\mathrm{mmHg})$ & $72(65-80)$ & $73(66-81)$ & $71(65-79)$ & $67(60-75)$ & $68(64-76)$ & $<0.01$ \\
\hline Use of statins, n (\%) & $266(36)$ & $177(32)$ & $55(47)$ & $16(34)$ & $18(47)$ & 0.02 \\
\hline Use of active vitamin $D_{3}, n(\%)$ & $55(7)$ & $30(5)$ & $17(15)$ & $2(4)$ & $6(16)$ & 0.01 \\
\hline Body mass index $\left(\mathrm{kg} / \mathrm{m}^{2}\right)$ & $22.9(20.5-25.1)$ & $22.9(20.6-25.5)$ & $23.0(20.7-24.7)$ & $23.0(19.8-25.3)$ & $22.2(20.9-24.0)$ & 0.28 \\
\hline Daily proteinuria (g) & $0.95(0.28-2.63)$ & $0.88(0.26-2.59)$ & $1.06(0.30-2.59)$ & $1.44(0.57-3.28)$ & $0.73(0.21-2.54)$ & 0.62 \\
\hline CRP (mg/L) & $1.0(0.5-2.0)$ & $0.9(0.5-2.0)$ & $0.9(0.5-2.0)$ & $1.2(0.6-2.0)$ & $1.3(0.5-2.9)$ & 0.16 \\
\hline eGFR (mL/min/1.73 $\left.\mathrm{m}^{2}\right)$ & $27.9(16.0-47.6)$ & $30.4(17.2-53.1)$ & $23.0(14.1-37.1)$ & $25.8(14.7-36.8)$ & $22.9(12.9-31.7)$ & $<0.01$ \\
\hline HDL-C (mmol/L) & $1.16(0.93-1.45)$ & $1.16(0.93-1.45)$ & $1.12(0.96-1.42)$ & $1.16(0.93-1.42)$ & $1.22(0.91-1.55)$ & 0.84 \\
\hline LDL-C (mmol/L) & $2.51(1.99-3.10)$ & $2.53(2.04-3.15)$ & $2.38(1.95-3.00)$ & $2.53(1.99-3.10)$ & $2.20(1.81-2.92)$ & 0.05 \\
\hline Serum albumin $(\mathrm{g} / \mathrm{L})$ & $34(30-38)$ & $35(31-38)$ & $34(29-36)$ & $31(25-35)$ & $33(30-36)$ & $<0.01$ \\
\hline Serum calcium (mmol/L) & $2.32(2.25-2.40)$ & $2.32(2.25-2.37)$ & $2.32(2.27-2.42)$ & $2.32(2.25-2.47)$ & $2.30(2.25-2.40)$ & 0.13 \\
\hline Serum phosphorus (mmol/L) & $1.19(1.07-1.32)$ & $1.19(1.07-1.32)$ & $1.19(1.07-1.36)$ & $1.16(1.07-1.29)$ & $1.26(1.13-1.39)$ & 0.28 \\
\hline PTH (pg/mL) & $62(43-102)$ & $60(40-100)$ & $64(43-107)$ & $74(55-116)$ & $65(43-102)$ & 0.24 \\
\hline $1,25(\mathrm{OH})_{2} \mathrm{D}(\mathrm{pg} / \mathrm{mL})$ & $28.9(20.4-39.6)$ & $30.2(21.2-40.5)$ & $25.2(18.6-35.5)$ & $29.0(20.4-37.7)$ & $24.1(16.7-35.8)$ & 0.01 \\
\hline FGF23 (pg/mL) & $83(57-130)$ & $79(54-125)$ & $89(60-152)$ & $89(70-132)$ & $102(71-158)$ & $<0.01$ \\
\hline LVH, n (\%) & $338(45)$ & $222(41)$ & $68(59)$ & $25(53)$ & $23(61)$ & $<0.01$ \\
\hline Carotid IMT (mm) & $0.70(0.60-0.82)$ & $0.68(0.58-0.80)$ & $0.75(0.66-0.87)$ & $0.75(0.69-0.83)$ & $0.76(0.67-0.86)$ & $<0.01$ \\
\hline CAP, n (\%) & $583(78)$ & $389(71)$ & $113(97)$ & $46(98)$ & $35(92)$ & $<0.01$ \\
\hline CAP score & $4.49(1.28-8.46)$ & $3.40(0.00-7.00)$ & $7.10(3.54-10.96)$ & $7.41(3.57-12.46)$ & $8.90(6.30-12.19)$ & $<0.01$ \\
\hline PAD, n (\%) & $123(16)$ & $65(12)$ & $28(24)$ & $15(32)$ & $15(39)$ & $<0.01$ \\
\hline ABPI & $1.07(1.00-1.14)$ & $1.08(1.02-1.15)$ & $1.05(0.95-1.13)$ & $1.07(0.90-1.16)$ & $0.95(0.89-1.08)$ & $<0.01$ \\
\hline \multicolumn{7}{|c|}{$\begin{array}{l}\text { Values are expressed as mean } \pm \text { SD, } n(\%) \text { or median (interquartile range). AVC, aortic valve calcification; IHD, ischemic heart disease; SBP, systolic blood } \\
\text { pressure; DBP, diastolic blood pressure; CRP, C-reactive protein; eGFR, estimated glomerular filtration rate; HDL-C, high-density lipoprotein cholesterol; LDL-C, } \\
\text { low-density lipoprotein cholesterol; PTH, parathyroid hormone; } 1,25(\mathrm{OH})_{2} \mathrm{D}, 1,25 \text {-dihydroxyvitamin } \mathrm{D} \text {; FGF23, fibroblast growth factor } 23 \text {; LVH, left } \\
\text { ventricular hypertrophy; IMT, intima-media thickness; CAP, carotid artery plaque; PAD, peripheral artery disease; ABPI, ankle-brachial blood pressure index. } \\
\text { *Trend analyses were performed across } 4 \text { groups ( } 1 \text { group without AVC and } 3 \text { groups with AVC). ** Ten patients had no available data. }\end{array}$} \\
\hline
\end{tabular}

The univariable logistic regression analyses are shown in Table 2. Older age, higher systolic blood pressure, and the presence of diabetes mellitus and ischemic heart disease were significantly associated with AVC. The use of statins and active vitamin $\mathrm{D}_{3}$ was associated with AVC. A lower eGFR and serum albumin level were related to AVC. As for the association between AVC and mineral metabolism, a higher serum calcium, lower log 1,25(OH) 2 D, and higher log FGF23 were related to AVC, whereas serum phosphorus and log PTH were not. Left ventricular hypertrophy was also associated with AVC. In addition, patients with CAP, PAD, higher CAP score, lower ABPI, and higher carotid IMT had a significantly higher risk of having AVC. We performed multivariable analyses to determine whether the presence of CAP, CAP score, the presence of PAD, ABPI, and carotid IMT were independently associated with AVC (Table 3). Adjusted for 
age and sex (model 1), the presence of CAP, higher CAP score, the presence of PAD, and a lower ABPI were all associated with AVC. Conversely, we found no significant association between AVC and carotid IMT. Similarly, in the fully adjusted model (model 4), the presence of CAP, higher CAP score, the presence of $\mathrm{PAD}$, and a lower ABPI, but not carotid IMT, were independent risk factors for AVC.

Table 2

Odds ratios for having AVC in the univariable analysis

\begin{tabular}{|c|c|c|c|}
\hline Variables & OR & $95 \% \mathrm{Cl}$ & $P$ \\
\hline Age (per 10-year increase) & 2.49 & $2.06-3.01$ & $<0.01$ \\
\hline Male & 1.29 & $0.91-1.83$ & 0.16 \\
\hline Diabetes mellitus & 1.58 & $1.14-2.19$ & $<0.01$ \\
\hline Smoking & 1.32 & $0.95-1.83$ & 0.10 \\
\hline Dyslipidemia & 1.00 & $0.69-1.44$ & 0.98 \\
\hline IHD & 2.28 & $1.50-3.48$ & $<0.01$ \\
\hline SBP (per 10-mmHg increase) & 1.14 & $1.04-1.25$ & $<0.01$ \\
\hline Body mass index (per $1-\mathrm{kg} / \mathrm{m}^{2}$ increase) & 0.99 & $0.95-1.02$ & 0.46 \\
\hline Log CRP (per 1-log unit increase) & 1.12 & $0.97-1.30$ & 0.12 \\
\hline Daily proteinuria (per 1-g increase) & 1.00 & $0.95-1.06$ & 0.92 \\
\hline eGFR (per 1-mL/min/1.73 m² increase) & 0.98 & $0.97-0.99$ & $<0.01$ \\
\hline Serum albumin (per 1-g/L increase) & 0.95 & $0.93-0.97$ & $<0.01$ \\
\hline Serum calcium (per 1-mmol/L increase) & 4.36 & $1.21-15.71$ & 0.02 \\
\hline Serum phosphorus (per 1-mmol/L increase) & 1.54 & $0.77-3.07$ & 0.22 \\
\hline Log PTH (per 1-log unit increase) & 1.04 & $0.83-1.31$ & 0.71 \\
\hline $\log 1,25(\mathrm{OH})_{2} \mathrm{D}$ (per 1-log unit increase) & 0.61 & $0.44-0.85$ & $<0.01$ \\
\hline Log FGF23 (per 1-log unit increase) & 1.44 & $1.18-1.77$ & $<0.01$ \\
\hline Use of statins & 1.67 & $1.20-2.32$ & $<0.01$ \\
\hline Use of active vitamin $D_{3}$ & 2.45 & $1.40-4.28$ & $<0.01$ \\
\hline LVH & 2.00 & $1.44-2.78$ & $<0.01$ \\
\hline CAP & 11.33 & $5.21-24.62$ & $<0.01$ \\
\hline CAP score (per 1.0-point increase) & 1.15 & $1.12-1.20$ & $<0.01$ \\
\hline PAD & 3.01 & $2.02-4.50$ & $<0.01$ \\
\hline ABPI (per 0.1-point increase) & 0.71 & $0.63-0.80$ & $<0.01$ \\
\hline Carotid IMT (per 0.1-mm increase) & 1.35 & $1.22-1.49$ & $<0.01$ \\
\hline \multicolumn{4}{|c|}{$\begin{array}{l}\text { AVC, aortic valve calcification; OR, odds ratio; } \mathrm{Cl} \text {, confidence interval; IHD, ischemic heart disease; SBP, systolic blood pressure; CRP, C-reactive protein: } \\
\text { eGFR, estimated glomerular filtration rate; PTH, parathyroid hormone; } 1,25(\mathrm{OH})_{2} \mathrm{D}, 1,25 \text {-dihydroxyvitamin } \mathrm{D} ; \mathrm{FGF} 23 \text {, fibroblast growth factor } 23 \text {; } \mathrm{LVH} \text {, left } \\
\text { ventricular hypertrophy; CAP, carotid artery plaque; PAD, peripheral artery disease; } \mathrm{ABPI} \text {, ankle-brachial blood pressure index; IMT, intima-media thickness }\end{array}$} \\
\hline
\end{tabular}


Table 3

Odds ratios for having AVC in the multivariable analyses

\begin{tabular}{|c|c|c|c|c|c|c|c|c|c|c|c|c|}
\hline & \multicolumn{3}{|c|}{ Model 1} & \multicolumn{3}{|c|}{ Model 2} & \multicolumn{3}{|c|}{ Model 3} & \multicolumn{3}{|c|}{ Model 4} \\
\hline & OR & $95 \% \mathrm{Cl}$ & $P$ & OR & $95 \% \mathrm{Cl}$ & $P$ & OR & $95 \% \mathrm{Cl}$ & $P$ & OR & $95 \% \mathrm{Cl}$ & $P$ \\
\hline CAP & 4.26 & $\begin{array}{l}1.87- \\
9.69\end{array}$ & $\dot{0.01}$ & 3.67 & $\begin{array}{l}1.60^{-} \\
8.43\end{array}$ & $\dot{0} 01$ & 3.47 & $\begin{array}{l}1.49- \\
8.10\end{array}$ & $\dot{0} 01$ & 3.37 & $\begin{array}{l}1.43^{-} \\
7.95\end{array}$ & 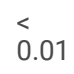 \\
\hline CAP score (per 1.0 increase) & 1.08 & $\begin{array}{l}1.04- \\
1.13\end{array}$ & $\dot{0.01}$ & 1.07 & $\begin{array}{l}1.03- \\
1.12\end{array}$ & $\dot{0} 0.01$ & 1.09 & $\begin{array}{l}1.02- \\
1.11\end{array}$ & $\begin{array}{l}< \\
0.01\end{array}$ & 1.06 & $\begin{array}{l}1.02- \\
1.11\end{array}$ & 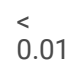 \\
\hline PAD & 1.95 & $\begin{array}{l}1.26- \\
3.01\end{array}$ & $\begin{array}{l}<.01 \\
0.01\end{array}$ & 1.73 & $\begin{array}{l}1.10- \\
2.71\end{array}$ & 0.02 & 1.77 & $\begin{array}{l}1.12- \\
2.80\end{array}$ & 0.02 & 1.76 & $\begin{array}{l}1.10- \\
2.81\end{array}$ & 0.02 \\
\hline ABPI (per 0.1 increase) & 0.80 & $\begin{array}{l}0.70- \\
0.91\end{array}$ & $\begin{array}{l}< \\
0.01\end{array}$ & 0.82 & $\begin{array}{l}0.72- \\
0.93\end{array}$ & $\begin{array}{l}< \\
0.01\end{array}$ & 0.83 & $\begin{array}{l}0.72- \\
0.95\end{array}$ & $\begin{array}{l}< \\
0.01\end{array}$ & 0.83 & $\begin{array}{l}0.72- \\
0.95\end{array}$ & $<0$ \\
\hline $\begin{array}{l}\text { Carotid IMT (per 0.1-mm } \\
\text { increase) }\end{array}$ & 1.07 & $\begin{array}{l}0.95- \\
1.21\end{array}$ & 0.25 & 1.05 & $\begin{array}{l}0.93- \\
1.19\end{array}$ & 0.43 & 1.03 & $\begin{array}{l}0.91- \\
1.17\end{array}$ & 0.62 & 1.02 & $\begin{array}{l}0.89- \\
1.16\end{array}$ & 0.80 \\
\hline \multicolumn{13}{|c|}{ Model 1: adjusted for age and sex. } \\
\hline \multicolumn{13}{|c|}{ Model 2: adjusted for model 1 plus smoking, diabetes mellitus, dyslipidemia, and systolic blood pressure. } \\
\hline \multicolumn{13}{|c|}{ Model 3: adjusted for model 2 plus daily proteinuria, eGFR, serum albumin, serum calcium, serum phosphorus, $\log$ PTH, $\log 1,25(\mathrm{OH})_{2} \mathrm{D}$, and $\log F G F 23}$. \\
\hline \multicolumn{13}{|c|}{ Model 4: adjusted for model 3 plus the presence of ischemic heart disease, body mass index, log CRP, use of statins, use of active vitamin $\mathrm{D}_{3}$ and LVH. } \\
\hline \multicolumn{13}{|c|}{ AVC, aortic valve calcification; OR, odds ratio; Cl, confidence interval; CAP, carotid artery plaque; PAD, peripheral artery disease; } \\
\hline \multicolumn{13}{|c|}{ ABPI, ankle-brachial blood pressure index; IMT, intima-media thickness; eGFR, estimated glomerular filtration rate; PTH, parathyroid hormone; } \\
\hline
\end{tabular}

We also investigated the adjusted ORs for having AVC according to subgroups stratified by demographic and clinical characteristics, as shown in Table 4. In male patients and patients without diabetes mellitus, the presence of CAP and PAD, the CAP score, and the ABPI were all associated with AVC. Conversely, none of the above four variables showed significant associations with AVC in female patients and patients with diabetes mellitus. With regard to interactions for having AVC between those four variables and the other baseline clinical characteristics, the $P$ values between CAP and the eGFR and among PAD, the eGFR, and the serum albumin level were $<0.1$; in contrast, the other interactions showed $P$ values of $>0.1$. 
Table 4

Adjusted odds ratios of CAP, CAP score, PAD, and ABPI for having AVC in subgroups stratified according to baseline characteristics

\begin{tabular}{|c|c|c|c|c|c|c|c|c|c|c|c|c|c|}
\hline \multirow[b]{2}{*}{ variables } & & \multirow[b]{2}{*}{$\begin{array}{l}\text { No. of } \\
\text { patients }\end{array}$} & \multirow[b]{2}{*}{$\begin{array}{l}\text { No. } \\
\text { of } \\
\text { AVC }\end{array}$} & \multicolumn{3}{|l|}{ CAP } & \multicolumn{3}{|c|}{$\begin{array}{l}\text { CAP score (per 1.0-point } \\
\text { increase) }\end{array}$} & \multicolumn{2}{|l|}{ PAD } & \multirow[b]{2}{*}{$\begin{array}{l}P \text { for } \\
\text { interaction }\end{array}$} & \multirow{2}{*}{$\frac{\mathrm{ABP}}{\mathrm{OR}}$} \\
\hline & & & & OR & $95 \% \mathrm{Cl}$ & $\begin{array}{l}P \text { for } \\
\text { interaction }\end{array}$ & OR & $95 \% \mathrm{Cl}$ & $\begin{array}{l}P \text { for } \\
\text { interaction }\end{array}$ & OR & $95 \% \mathrm{Cl}$ & & \\
\hline \multirow[t]{2}{*}{ Age (years) } & $\begin{array}{l}\mathrm{L}(< \\
69.8)\end{array}$ & 375 & 44 & 16.79 & $\begin{array}{l}{ }^{2} 2.07- \\
136.4\end{array}$ & 0.23 & 1.02 & $\begin{array}{l}0.94- \\
1.12\end{array}$ & 0.16 & 2.72 & $\begin{array}{l}0.91- \\
8.15\end{array}$ & 0.91 & 0.90 \\
\hline & $\begin{array}{l}H(> \\
69.8)\end{array}$ & 374 & 157 & 1.94 & $\begin{array}{l}0.70- \\
5.37\end{array}$ & & 1.09 & $\begin{array}{l}\mathrm{a} 1.03- \\
1.15\end{array}$ & & 1.84 & $\begin{array}{l}\mathrm{b} 1.07- \\
3.15\end{array}$ & & 0.78 \\
\hline \multirow[t]{2}{*}{ Sex } & Male & 499 & 142 & 3.55 & $\begin{array}{l}b_{1} 1.00- \\
12.52\end{array}$ & 0.96 & 1.07 & $\begin{array}{l}\mathrm{a} 1.02- \\
1.13\end{array}$ & 0.82 & 2.04 & $\begin{array}{l}\mathrm{b}_{1} 1.17- \\
3.54\end{array}$ & 0.58 & 0.79 \\
\hline & Female & 250 & 59 & 3.12 & $\begin{array}{l}0.93- \\
10.39\end{array}$ & & 1.06 & $\begin{array}{l}0.97- \\
1.17\end{array}$ & & 1.04 & $\begin{array}{l}0.36- \\
3.01\end{array}$ & & 0.96 \\
\hline \multirow[t]{2}{*}{$\begin{array}{l}\text { eGFR } \\
\left(\mathrm{mL} / \mathrm{min} / 1.73 \mathrm{~m}^{2}\right)\end{array}$} & $\begin{array}{l}\mathrm{L}(< \\
28.0)\end{array}$ & 375 & 126 & 1.26 & $\begin{array}{l}0.43- \\
3.72\end{array}$ & 0.06 & 1.06 & $\begin{array}{l}\mathrm{b}_{1} 1.00- \\
1.12\end{array}$ & 0.40 & 1.62 & $\begin{array}{l}0.90- \\
2.93\end{array}$ & 0.049 & 0.78 \\
\hline & $\begin{array}{l}\mathrm{H}(> \\
28.0)\end{array}$ & 374 & 75 & 7.76 & $\begin{array}{l}\text { a } 1.70- \\
35.47\end{array}$ & & 1.08 & $\begin{array}{l}0.999- \\
1.17\end{array}$ & & 1.94 & $\begin{array}{l}0.78- \\
4.77\end{array}$ & & 0.91 \\
\hline \multirow[t]{2}{*}{ DM } & Absence & 466 & 109 & 4.91 & $\begin{array}{l}\text { a } 1.57- \\
15.34\end{array}$ & 0.11 & 1.10 & $\begin{array}{l}\mathrm{a} 1.03- \\
1.17\end{array}$ & 0.09 & 2.17 & $\begin{array}{l}b_{1} 1.03- \\
4.58\end{array}$ & 0.48 & 0.74 \\
\hline & Presence & 283 & 92 & 1.31 & $\begin{array}{l}0.32- \\
5.44\end{array}$ & & 1.03 & $\begin{array}{l}0.96- \\
1.11\end{array}$ & & 1.41 & $\begin{array}{l}0.72- \\
2.76\end{array}$ & & 0.89 \\
\hline \multirow[t]{2}{*}{ S-Alb (g/L) } & $L(\leq 34)$ & 383 & 128 & 4.71 & $\begin{array}{l}\mathrm{b}_{1} 1.27- \\
17.47\end{array}$ & 0.13 & 1.06 & $\begin{array}{l}\mathrm{b}_{1} 1.00- \\
1.12\end{array}$ & 0.21 & 2.45 & $\begin{array}{l}\mathrm{a} 1.30- \\
4.60\end{array}$ & 0.08 & 0.81 \\
\hline & $\mathrm{H}(>34)$ & 366 & 73 & 1.54 & $\begin{array}{l}0.46- \\
5.24\end{array}$ & & 1.12 & $\begin{array}{l}\mathrm{b}_{1} .02- \\
1.22\end{array}$ & & 1.04 & $\begin{array}{l}0.44- \\
2.48\end{array}$ & & 0.85 \\
\hline
\end{tabular}

CAP, carotid artery plaque; PAD, peripheral artery disease; $\mathrm{ABPI}$, ankle-brachial blood pressure index; $\mathrm{AVC}$, aortic valve calcification; OR, odds ratio; $\mathrm{Cl}$, confiden high; eGFR, estimated glomerular filtration rate; DM, diabetes mellitus; S-Alb, serum albumin. Adjusted for the same covariates in Model 4 in Table 3 . ${ }^{a} P<0.01$

\section{Discussion}

The present study explored the factors associated with AVC in overall patients with CKD not on dialysis. The prevalence of AVC in our cohort was similar to that in previous reports $[10,23]$. In the multivariable analysis, the presence of CAP and PAD, the CAP score, and ABPI were independent risk factors for AVC beyond traditional and nontraditional CV risk factors. In addition, the stratified analyses demonstrated that AVC was independently associated with any of the four variables of $C A P, P A D$, the CAP score, or the ABPI in all subgroups other than female patients and patients with diabetes mellitus.

The pathological mechanisms for the development of AVC are now believed to constitute an active cellular process that develops within the aortic valve leaflets, following mechanisms similar to atherosclerosis with lipoprotein deposition, chronic inflammation, and active leaflet calcification [28-30]. A previous population-based study showed that AVC was associated with a high incidence of atherosclerotic risk factors, suggesting that AVC is a manifestation of systemic atherosclerosis [15]. In patients with CKD, including dialysis patients, combined aortic and mitral valve calcification has also been regarded as a marker of the systemic atherosclerotic process $[18,25]$. The present study was conducted in patients with CKD not on dialysis and also showed independent associations between AVC and subclinical atherosclerotic lesions such as CAP and PAD. These findings might suggest that AVC and atherosclerosis share common pathological mechanism and/or risk factors.

Several studies have addressed the association between kidney function and the prevalence of AVC. However, this relationship has remained unclear. A previous report showed a significant association of AVC with the presence of CKD; predialysis patients with CKD were approximately three times more likely to have AVC than patients without CKD [21]. In contrast, a large cohort study showed no significant association between the presence of CKD and the prevalence of AVC [39]. Another large cohort study also failed to show a significant association of AVC with impaired kidney function in the multivariable analysis [16]. The Chronic Renal Insufficiency Cohort (CRIC) Study demonstrated that decreased kidney function was significantly associated with AVC in the multivariable analysis after adjustment for traditional CV risk factors, but the relationship disappeared after adding nontraditional CV risk factors including high sensitivity C-reactive protein and plasma homocysteine as covariates [40]. In our cohort, the prevalence of AVC significantly increased with advancing CKD stages, and AVC was related to the eGFR in the univariable analysis; however, the multivariable analysis (model 4) did not show an association between AVC and the eGFR (data not shown). Furthermore, Piers et al. [22] noted that AVC was more frequent and severe in patients with atherosclerotic causes of CKD than in those with non-atherosclerotic causes of CKD, suggesting that AVC is associated with the atherosclerotic burden rather than the severity of CKD.

The association between AVC and derangement of mineral metabolism has been documented in several studies. Deranged mineral metabolism occurs with advancing CKD stages. One study showed that AVC was associated with higher FGF23 and PTH levels in patients with mild to moderate CKD [41], whereas a different population-based study showed no significant association of AVC with FGF23 or PTH [14]. In dialysis patients, the calcium $\times$ phosphate product has been shown to be a significant risk factor for AVC [42]. In population-based studies, the serum phosphorus level was also positively associated with the

Page $7 / 11$ 
prevalence and incidence of AVC $[13,14]$. Conversely, each $0.32-\mathrm{mmol} / \mathrm{L}$ increment in the serum phosphorus level in patients with CKD was associated with coronary artery and mitral valve calcification, but not with AVC [23]. Furthermore, the CRIC Study revealed that the serum calcium, serum phosphorus, and PTH levels were not independently associated with AVC [40]. The present study also showed significant associations of AVC with markers of mineral metabolism (serum calcium, $\log 1,25(\mathrm{OH})_{2} \mathrm{D}$, and $\log$ FGF23) in the univariable analyses, but not in the multivariable analyses (model 4) (data not shown). Based on these findings, the substantial effects of derangement of mineral metabolism on the development of AVC have remained controversial, and further investigations are warranted to elucidate the association between AVC and deranged mineral metabolism.

In the present study, univariable logistic analysis revealed no significant association between AVC and male sex (Table 2). Furthermore, in the stratified analyses, we found no significant interactions between sex and parameters such as the presence of CAP, CAP score, the presence of PAD, and ABPI on AVC. However, each of these atherosclerotic parameters was associated with AVC in men, but not in women, although the reason for the lack of association of AVC with these parameters in women remains unclear.

\section{Study Limitations}

The present study has some limitations. First, all patients were recruited at a single regional hospital; thus, the selection of patients was limited and the sample size was relatively small. Second, our cohort had an imbalanced sex ratio and was relatively old, probably because of the recruitment of consecutive patients who were admitted to our hospital. Third, this cross-sectional study did not allow us to draw causal relationships. Fourth, the clinical data including the echocardiographic findings were recorded at one study time. Fifth, we did not evaluate fetuin-A, matrix-Gla-protein, reactive oxygen species, asymmetric dimethylarginine, or receptor activator of nuclear factor-KB ligand, all of which can play roles in aortic valve calcific degeneration associated with CKD [32]. Finally, we did not evaluate AVC quantitatively. AVC score quantitatively measured by computed tomography can detect valve calcification with higher sensitivity [43]; accordingly, the echocardiographic AVC evaluation method that we used is considered inferior to that with computed tomography. In this study, we performed multivariable logistic regression analyses, adjusted for several covariates, particularly markers of deranged mineral metabolism such as serum calcium, serum phosphorus, $\log \mathrm{PTH}, \log 1,25(\mathrm{OH})_{2} \mathrm{D}$, and $\log \mathrm{FGF} 23$, which were measured simultaneously. Accordingly, despite the limitations, our results may indicate a robust relationship between AVC and atherosclerotic parameters.

\section{Conclusions}

The present study showed that AVC was associated with the presence of CAP and PAD, and the CAP score and ABPI, independent of traditional and nontraditional CV risk factors. We consider that the association between AVC and these subclinical atherosclerotic lesions might reflect the burden of shared atherosclerotic risk factors.

\section{Abbreviations}

CKD: chronic kidney disease; CV: cardiovascular; AVC: aortic valve calcification; CAP: carotid artery plaque; PAD: peripheral artery disease; IMT: intima-media thickness; ABPI: ankle-brachial blood pressure index; FGF23: fibroblast growth factor 23; PTH: parathyroid hormone; 1,25(OH) 2 D: 1,25-dihydroxyvitamin D; eGFR: estimated glomerular filtration rate; OR: odds ratio; Cl: confidence interval; CRIC: Chronic Renal Insufficiency Cohort.

\section{Declarations}

\section{Ethics approval and consent to participate}

This study was approved by the Ethics Committee of the National Hospital Organization Kyushu Medical Center (Approval Number: 09-09). Informed written consent was obtained from all participants included in this study.

\section{Consent for publication}

Not applicable.

\section{Availability of data and materials}

The data used and analyzed in this study are available from the corresponding author on reasonable request.

\section{Competing interests}

The authors declare that they have no competing interests.

\section{Funding}

Not applicable.

\section{Authors' contributions}

YA and MN collected and analyzed data and wrote the manuscript. YM and RY collected data and contributed to discussions. MS, AF, ST, YS, RI, KA, and MT collected data and substantially contributed to the data interpretation. TN, KT, and TK substantially contributed to the data interpretation and reviewed and revised the manuscript. All authors read and approved the final manuscript. 


\section{Acknowledgments}

We would like to express our gratitude to the 749 participants of our study and to the cardiologists who assessed the cardiac structures on the echocardiograms. We also thank Angela Morben, DVM, ELS, from Edanz Group (www.edanzediting.com/ac), for editing a draft of this manuscript.

\section{References}

(1) Herzog CA, Asinger RW, Berger AK, Charytan DM, Di'ez J, Hart RG, et al. Cardiovascular disease in chronic kidney disease. A clinical update from Kidney Disease: Improving Global Outcomes (KDIGO). Kidney Int. 2011; 80: 572-86.

(2) Samad Z, Sivak JA, Phelan M, Schulte PJ, Patel U, Velazquez EJ. Prevalence and Outcomes of Left-Sided Valvular Heart Disease Associated With Chronic Kidney Disease. J Am Heart Assoc. 2017; 6: e006044.

(3) Sarnak MJ, Levey AS, Schoolwerth AC, Coresh J, Culleton B, Hamm LL, et al.; American Heart Association Councils on Kidney in Cardiovascular Disease, High Blood Pressure Research, Clinical Cardiology, and Epidemiology and Prevention. Kidney disease as a risk factor for development of cardiovascular disease: a statement from the American Heart Association Councils on Kidney in Cardiovascular Disease, High Blood Pressure Research, Clinical Cardiology, and Epidemiology and Prevention. Circulation. 2003;108:2154-69.

(4) Go AS, Chertow GM, Fan D, McCulloch CE, Hsu CY. Chronic kidney disease and the risks of death, cardiovascular events, and hospitalization. N Engl J Med. 2004;351:1296-305.

(5) Clavel MA, Pibarot P, Messika-Zeitoun D, Capoulade R, Malouf J, Aggarval S, et al. Impact of aortic valve calcification, as measured by MDCT, on survival in patients with aortic stenosis: results of an international registry study. J Am Coll Cardiol. 2014; 64:1202-13.

(6) Thomassen HK, Cioffi G, Gerdts E, Einarsen E, Midtbø HB, Mancusi C, et al. Echocardiographic aortic valve calcification and outcomes in women and men with aortic stenosis. Heart. 2017; 103:1619-24.

(7) Owens DS, Budoff MJ, Katz R, Takasu J, Shavelle DM, Carr JJ, et al. Aortic valve calcium independently predicts coronary and cardiovascular events in a primary prevention population. JACC Cardiovasc Imaging. 2012; 5: 619-25.

(8) Blaha MJ, Budoff MJ, Rivera JJ, Khan AN, Santos RD, Shaw LJ, et al. Relation of aortic valve calcium detected by cardiac computed tomography to allcause mortality. Am J Cardiol. 2010; 106: 1787-91.

(9) Raggi P, Bellasi A, Gamboa C, Ferramosca E, Ratti C, Block GA, et al. All-cause mortality in hemodialysis patients with heart valve calcification. Clin J Am Soc Nephrol. 2011; 6:1990-5.

(10) Hensen LCR, Mahdiui ME, van Rosendael AR, Smit JM, Jukema JW, Bax JJ, et al. Prevalence and Prognostic Implications of Mitral and Aortic Valve Calcium in Patients With Chronic Kidney Disease. Am J Cardiol. 2018; 122: 1732-7.

(11) Owens DS, Budoff MJ, Katz R, Takasu J, Shavelle DM, Carr JJ, et al. Aortic valve calcium independently predicts coronary and cardiovascular events in a primary prevention population. JACC Cardiovasc Imaging. 2012; 5: 619-25.

(12) Hoffmann U, Massaro JM, D'Agostino RB Sr, Kathiresan S, Fox CS, O'Donnell CJ. Cardiovascular Event Prediction and Risk Reclassification by Coronary, Aortic, and Valvular Calcification in the Framingham Heart Study. J Am Heart Assoc. 2016; 5: e003144.

(13) Hisamatsu T, Miura K, Fujiyoshi A, Kadota A, Miyagawa N, Satoh A, et al.; SESSA Research Group. Serum magnesium, phosphorus, and calcium levels and subclinical calcific aortic valve disease: A population-based study. Atherosclerosis. 2018; 273: 145-52.

(14) Linefsky JP, O'Brien KD, Sachs M, Katz R, Eng J, Michos ED, et al. Serum phosphate is associated with aortic valve calcification in the Multi-ethnic Study of Atherosclerosis (MESA). Atherosclerosis. 2014; 233: 331-7.

(15) Boon A, Cheriex E, Lodder J, Kessels F. Cardiac valve calcification: characteristics of patients with calcification of the mitral annulus or aortic valve. Heart. 1997; 78: 472-4.

(16) Ix JH, Shlipak MG, Katz R, Budoff MJ, Shavelle DM, Probstfield JL, et al. Kidney function and aortic valve and mitral annular calcification in the MultiEthnic Study of Atherosclerosis (MESA). Am J Kidney Dis. 2007; 50: 412-20.

(17) Ribeiro S, Ramos A, Brandão A, Rebelo JR, Guerra A, Resina C, et al. Cardiac valve calcification in haemodialysis patients: role of calcium-phosphate metabolism. Nephrol Dial Transplant. 1998; 13: 2037-40.

(18) Wang AY, Ho SS, Wang M, Liu EK, Ho S, Li PK, et al. Cardiac valvular calcification as a marker of atherosclerosis and arterial calcification in end-stage renal disease. Arch Intern Med. 2005; 165: 327-32.

(19) Takahashi H, Ishii H, Aoyama T, Kamoi D, Kasuga H, Ito Y, et al. Association of cardiac valvular calcifications and C-reactive protein with cardiovascular mortality in incident hemodialysis patients: a Japanese cohort study. Am J Kidney Dis. 2013; 61: 254-61. 
(20) Ikee R, Honda K, Ishioka K, Oka M, Maesato K, Moriya H, et al. Differences in associated factors between aortic and mitral valve calcification in hemodialysis. Hypertens Res. 2010; 33: 622-6.

(21) Kim IY, Kim MJ, Lee DW, Lee SB, Shin MJ, Rhee H, et al. Cardiac valve calcification is associated with presence and severity of coronary artery disease in patients with pre-dialysis chronic kidney disease. Clin Exp Nephrol. 2015; 19: 1090-7

(22) Piers LH, Touw HR, Gansevoort R, Franssen CF, Oudkerk M, Zijlstra F, et al. Relation of aortic valve and coronary artery calcium in patients with chronic kidney disease to the stage and etiology of the renal disease. Am J Cardiol. 2009; 103: 1473-7.

(23) Adeney KL, Siscovick DS, Ix JH, Seliger SL, Shlipak MG, Jenny NS, et al. Association of serum phosphate with vascular and valvular calcification in moderate CKD. J Am Soc Nephrol. 2009; 20: 381-7.

(24) Stewart BF, Siscovick D, Lind BK, Gardin JM, Gottdiener JS, Smith VE, et al. Clinical factors associated with calcific aortic valve disease. Cardiovascular Health Study. J Am Coll Cardiol. 1997; 29: 630-4.

(25) Leskinen Y, Paana T, Saha H, Groundstroem K, Lehtimäki T, Kilpinen S, et al. Valvular calcification and its relationship to atherosclerosis in chronic kidney disease. J Heart Valve Dis. 2009; 18: 429-38.

(26) Hirata T, Arai Y, Takayama M, Abe Y, Ohkuma K, Takebayashi T. Carotid Plaque Score and Risk of Cardiovascular Mortality in the Oldest Old: Results from the TOOTH Study. J Atheroscler Thromb. 2018; 25: 55-64.

(27) Hamburg NM, Creager MA. Pathophysiology of Intermittent Claudication in Peripheral Artery Disease. Circ J. 2017; 81: 281-9.

(28) Mohler ER 3rd. Mechanisms of aortic valve calcification. Am J Cardiol. 2004; 94: 1396-402.

(29) Freeman RV, Otto CM. Spectrum of calcific aortic valve disease: pathogenesis, disease progression, and treatment strategies. Circulation. 2005; 111: $3316-26$.

(30) Small A, Kiss D, Giri J, Anwaruddin S, Siddiqi H, Guerraty M, et al. Biomarkers of Calcific Aortic Valve Disease. Arterioscler Thromb Vasc Biol. 2017; 37: 623-32.

(31) Sathyamurthy I, Alex S. C alcific aortic valve disease: is it another face of atherosclerosis? Indian Heart J. 2015; 67: 503-6.

(32) Rattazzi M, Bertacco E, Del Vecchio A, Puato M, Faggin E, Pauletto P. Aortic valve calcification in chronic kidney disease. Nephrol Dial Transplant. 2013; 28: $2968-76$

(33) Matsuo S, Imai E, Horio M, Yasuda Y, Tomita K, Nitta K, et al.; Collaborators developing the Japanese equation for estimated GFR: Revised equations for estimated GFR from serum creatinine in Japan. Am J Kidney Dis. 2009; 53: 982-92.

(34) Kidney Disease: Improving Global Outcomes (KDIGO) CKD Work Group. KDIGO 2012 clinical practice guideline for the evaluation and management of chronic kidney disease. Kidney Int Suppl. 2013;3:S1-150.

(35) Yoshitomi R, Nakayama M, Ura Y, Kuma K, Nishimoto H, Fukui A, et al. Ankle-brachial blood pressure index predicts cardiovascular events and mortality in Japanese patients with chronic kidney disease not on dialysis. Hypertens Res. 2014; 37: 1050-5.

(36) Naqvi AZ, Davis RB, Mukamal KJ. Nutrient intake and peripheral artery disease in adults: key considerations in cross-sectional studies. Clin Nutr. 2014; 33: 443-7.

(37) Sumida Y, Nakayama M, Nagata M, Nakashita S, Suehiro T, Kaizu Y, et al. Carotid artery calcification and atherosclerosis at the initiation of hemodialysis in patients with end-stage renal disease. Clin Nephrol. 2010; 73: 360-9.

(38) Yoshitomi R, Fukui A, Nakayama M, Ura Y, Ikeda H, Oniki H, et al. Sex differences in the association between serum uric acid levels and cardiac hypertrophy in patients with chronic kidney disease. Hypertens Res. 2014; 37: 246-52.

(39) Fox CS, Larson MG, Vasan RS, Guo CY, Parise H, Levy D, et al. Cross-sectional association of kidney function with valvular and annular calcification: the Framingham heart study. J Am Soc Nephrol. 2006; 17: 521-7.

(40) Guerraty MA, Chai B, Hsu JY, Ojo AO, Gao Y, Yang W, et al.; CRIC Study Investigators. Relation of aortic valve calcium to chronic kidney disease (from the Chronic Renal Insufficiency Cohort Study). Am J Cardiol. 2015; 115: 1281-6.

(41) Di Lullo L, Gorini A, Bellasi A, Morrone LF, Rivera R, Russo L, et al. Fibroblast growth factor 23 and parathyroid hormone predict extent of aortic valve calcifications in patients with mild to moderate chronic kidney disease. Clin Kidney J. 2015; 8: 732-6.

(42) Maher ER, Young G, Smyth-Walsh B, Pugh S, Curtis JR. Aortic and mitral valve calcification in patients with end-stage renal disease. Lancet. 1987; 2: 8757.

(43) Messika-Zeitoun D, Aubry MC, Detaint D, Bielak LF, Peyser PA, Sheedy PF, et al. Evaluation and clinical implications of aortic valve calcification measured by electron-beam computed tomography. Circulation. 2004;110:356-62.

Page 10/11 


\section{Additional Files}

Additional file 1: Supplementary materials 1. Correlation between AVC score and CAP score. The dashed lines indicate $95 \%$ confidence intervals. $\mathrm{R}^{2}=0.097$, coefficient of correlation $=1.868, P<0.01$. AVC, aortic valve calcification; CAP, carotid artery plaque

Additional file 2: Supplementary materials 2. Correlation between AVC score and ABPI. The dashed lines indicate $95 \%$ confidence intervals. $\mathrm{R}^{2}=0.048$, coefficient of correlation $=-0.036, P<0.01$. AVC, aortic valve calcification; ABPI, ankle-brachial blood pressure index

Additional file 3: Supplementary materials 3. Correlation between AVC score and eGFR. The dashed lines indicate $95 \%$ confidence intervals. $R^{2}=0.027$, coefficient of correlation $=-5.064, P<0.01$. AVC, aortic valve calcification; eGFR, estimated glomerular filtration rate

\section{Supplementary Files}

This is a list of supplementary files associated with this preprint. Click to download.

- BMCnephrologyAdditionalfile1Arita.pptx

- BMCnephrologyAdditionalfile3Arita.pptx

- BMCnephrologyAdditionalfile2Arita.pptx 